\title{
Influence of Blade Number on the Flow Characteristics in the Vertical Axis Propeller Hydro Turbine
}

\author{
Sun-Seok Byeon $^{1}$ and Youn-Jea Kim ${ }^{2}$ \\ ${ }^{1}$ Graduate School of Mechanical Engineering, Sungkyunkwan University \\ 300 Cheoncheon-dong, Suwon, 440-746, Korea, mildstone@skku.edu \\ ${ }^{2}$ School of Mechanical Engineering, Sungkyunkwan University \\ 300 Cheoncheon-dong, Suwon, 440-746, Korea, yjkim@skku.edu
}

\begin{abstract}
In this paper, the design method of a low-head propeller-type hydro turbine is studied for various numbers of blades on an axial propeller. We also investigate the relationship between geometrical parameters and internal performance parameters, such as angular velocities (100, 200, 300, $400 \mathrm{rpm})$ and 2.5 4m low heads through a three-dimensional numerical method with the SST turbulent model. The numerical results showed that the blade number had a more dominant influence than the change in heads and rotational speed on the flow characteristics of the turbine. The distributions of pressure and velocity in the streamwise direction of the propeller turbine were graphically depicted. Especially, the relationship among dimensionless parameters like specific speed $\left(\mathrm{N}_{\mathrm{s}}\right)$, flow coefficient $(\phi)$ and power coefficient ( $P$ ) were investigated.
\end{abstract}

Keywords: Propeller hydro turbine, Free vortex theory, Shaft power, Head, Blade number.

\section{Introduction}

\subsection{Background}

Small hydro power is power derived from the potential energy of falling water, which may generate electricity via the rotary power of a hydraulic turbine. Among the common types of renewable energy, hydroelectricity is the most actually used. However, it has been difficult to generate small hydro power in Korea compared with other renewable energies such as solar energy, wind energy, etc. due to its low economics and seasonal dependence. With the appropriate blade number, the propeller turbine can achieve high efficiency. Hence, it has wide application in appropriate conditions and economic feasibility.

Recent research has focused on the low head small hydro turbine due to the lack of natural resources having high head conditions and challenging boundary conditions like large variations of flow and optimum power generation [1]. Thus, it would be meaningful to predict the inner flow characteristics of a hydraulic water turbine by investigating dimensionless parameters such as specific speed $\left(\mathrm{N}_{\mathrm{s}}\right)$, power coefficient ( $P$ ), and flow coefficient $(\phi)$, etc.

Singh and Nestmann [2,3] investigated the influences of design parameters like the blade profile, blade height and blade number on the performance of a low head axial flow turbine for micro-hydro applications and examined the functionality of the internal performance parameters of the runner. They concluded that the blade number is a more dominating parameter than the blade height, although its variation did not result in major performance gains. They also investigated the exit tip modification on all three runner stages consistently and showed that the reduction of the exit tip blade angle increases the flow and output shaft power and positively affects the turbine efficiency at constant speed and head under the partial load operating condition. Also, Singh and Nestmann [4] found that the operating point of an experimentally optimized runner slightly deviated from the specifications of the free vortex design. This deviation was attributed to a probable variation of the absolute flow angle at the entry to the runner by the exit guide vane angle of $45^{\circ}$ and a small variation with respect to the runner geometry itself. Yassi and Hashemloo [5] deduced that the performance of the Agnew turbine can be improved for all part load conditions by adding guide vanes and a second rest point to its main shaft.

Ferro et al. [6] designed the rotor blades of a mini hydraulic bulb-turbine using the through-flow analysis approach, combining the streamline curvature method to solve for the meridional flow and the panel method to solve for the blade-to-blade flow. Using the commercial CFD tool, the rotor blades produced the desired turbine head, approximately, at the design condition of zero

Received September 11 2012; revised 2January 21 2013; accepted for publication February 28 2013: Review conducted by Prof. Hyung-Hee Cho. (Paper number R13009S)

Corresponding author: Youn-Jea Kim, Professor, yjkim@skku.edu 
angular momentum at the exit section. Alexander et al. [7] designed and built various turbines at one scale and presented practical, working and economic turbine solutions for low head microhydro systems. They also studied theoretical models, which showed how these flat blades can be closely approach the ideal blade shapes.

In this paper, steady three-dimensional turbulent flow throughout the whole flow passage of a propeller turbine is simulated using CFX Ver. 13 software. With respect to variables such as angular velocity, head, blade number, it is important to analyze the flow field of a hydraulic turbine and deduce dimensionless parameters to compare their correlations with these variables for different boundary conditions

\subsection{Technical objectives}

(i) To analyze the internal hydraulics effect due to changes in a parameter like angular velocity.

(ii) To analyze the effect of an internal variable through the dimensionless parameters of a hydraulic turbine.

(iii) To consider the overall performance of a small hydro turbine and the characteristics of inner flow.

\section{Theoretical background}

\subsection{Specific speed}

To design a hydraulic turbine, some preliminary design data, such as head $H$, volume flow rate $Q$, rotational speed $N$, and a non-dimensional parameter called the specific speed, $N_{\mathrm{s}}$, are needed. This design parameter is used to choose the appropriate hydraulic turbine for a location condition. This non-dimensional parameter is defined in Eq. (1) [8].

$$
N_{s}=\frac{\phi^{1 / 2}}{\psi^{3 / 4}}=\frac{N Q^{1 / 2}}{(g H)^{3 / 4}}
$$

By dimensional analysis, the three parameters such as $g H, \eta, P$, also called net energy transfer coefficients, reduce to the following equations [8]:

$$
\begin{aligned}
& \psi=\frac{g H}{(N D)^{2}}=f_{1}\left(\frac{Q}{N D^{3}}, \frac{\rho N D^{2}}{\mu}, \frac{l_{1}}{D}, \ldots\right) \\
& P=\frac{P}{\rho N^{3} D^{5}}=f_{2}\left(\frac{Q}{N D^{3}}, \frac{\rho N D^{2}}{\mu}, \frac{l_{1}}{D}, \ldots\right)
\end{aligned}
$$

Equations (2) (3) are the energy transfer coefficients, specifically, head coefficient for Eq. (2) and power coefficient for Eq. (3). The non-dimensional group $\left(Q /\left(N D^{3}\right)=\phi\right.$ ) is a volumetric flow coefficient and $\rho N D^{2} / \mu$ is a form of Reynolds number, Re.

\subsection{Free vortex theory}

Dixon [8] and Gorla et al. [9] presented a general free vortex theory for axial flow turbomachinery. Velocity triangles vary from the root to the tip of the blade because the blade speed $U$ is not constant and varies from root to tip. Thus, a twisted blading designed to take account of the changing angles is called vortex blading. For an axial hydraulic water turbine, the momentum equation is given by Eq. (4).

$$
\frac{1}{\rho} \frac{d P}{d r}=\frac{C_{w}^{2}}{r}
$$

The free vortex law can be derived from the momentum equation. For constant axial velocity, the whirl velocity $C_{w}$ is inversely proportional to the radius, which is given by Eq. (5).

$$
C_{w} \times r=\text { constant }
$$

That is, the whirl component of the velocity, $C_{w}$, and the radius are constant all along the inlet region and the exit region of the blade, and thus, the blade angle can be designed with respect to the radius vector. To maximize the energy transfer at the exit of the runner, the exit whirl velocity, $C_{\text {exit }}$ is zero and the axial velocity is constant.

Figure 1 shows the velocity diagram of the propeller hydraulic turbine drawn at the hub. Figure 2 represents the configuration of the runner blade. The runner design is developed for a gross head of $3.5 \mathrm{~m}$ and flowrate of $11.2 \mathrm{~m}^{3} / \mathrm{s}$. 


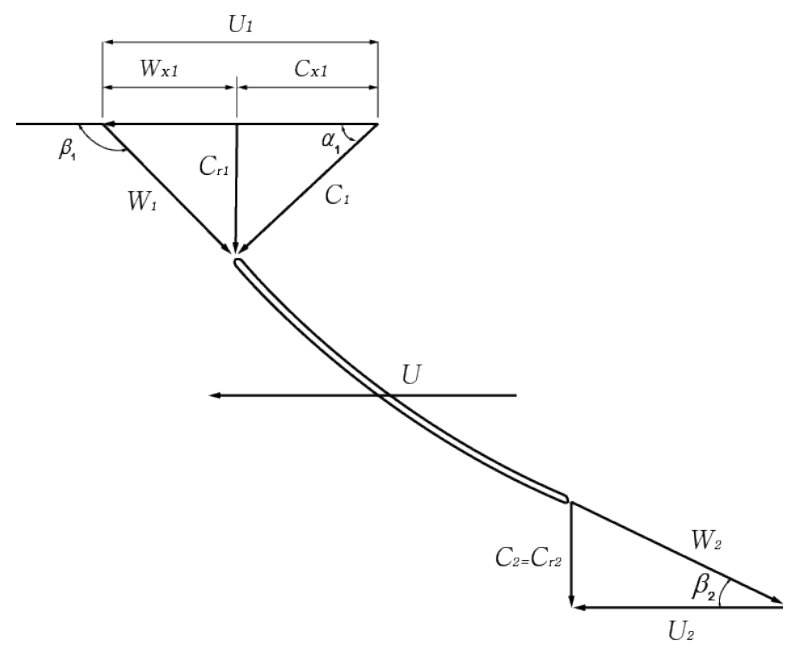

Fig. 1 Inlet and exit velocity triangles at the runner hub

[1].

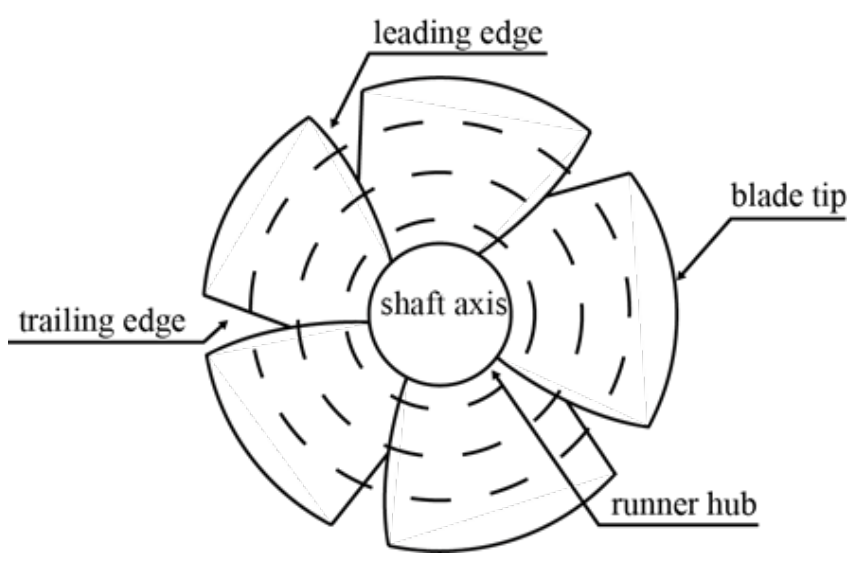

Fig. 2 Configuration of the runner blade [1].

\section{Numerical analysis}

\subsection{Governing equations}

The instantaneous conservation equations of continuity and momentum are as follows:

(i) continuity:

$$
\frac{\partial(\rho \vec{U})}{\partial t}+\nabla \cdot(\rho \vec{U})=0
$$

(ii) momentum:

$$
\frac{\partial(\rho \vec{U})}{\partial t}+\nabla \cdot(\rho \vec{U} \vec{U})=-\nabla p+\nabla \cdot \tau
$$

In order to analyze the inner flow of the axial propeller turbine, the SST (Shear Stress Turbulence) model was applied in this study. The $k-\omega$ equation based on the SST model accounts for the transport of the turbulent shear stress and gives highly accurate predictions of the onset and the amount of flow separation under adverse pressure gradients.

The BSL (Baseline $k-\omega$ ) model combines the advantages of the Wilcox and the $k-\omega$ model [10], but still fails to properly predict the onset and amount of flow separating from the smooth surfaces. The reasons for this deficiency are given in detail by Menter [11]. The main reason is that both models do not account for the transport of the turbulent shear stress. This model over predicts the eddy-viscosity. The proper transport behavior can be obtained by a limiter that is applied to the formulation of the eddy-viscosity: 


$$
\begin{gathered}
v_{t}=\frac{a_{1} k}{\max \left(a_{1} \omega, S F_{2}\right)} \\
v_{t}=\frac{\mu_{t}}{\rho}
\end{gathered}
$$

where, $F_{2}$ is a blending function similar to $F_{1}$, which restricts the limiter to the wall boundary layer, as the underlying assumptions are not correct for free shear flows, and $S$ denotes an invariant measure of the strain rate. The blending functions, which are critical to the success of the method, are formulated based on the distance to the nearest surface and on the flow variables [12].

$$
\begin{gathered}
F_{1}=\tanh \left(\arg _{1}^{4}\right) \\
\arg _{1}=\min \left(\max \left(\frac{\sqrt{k}}{\beta^{\prime} \omega y^{\prime}}, \frac{500 v}{y^{2} \omega}\right), \frac{4 \rho k}{C D_{k w} \sigma_{\omega 2} y^{2}}\right)
\end{gathered}
$$

where $y^{\prime}$ is the distance to the nearest wall, $v$ is the kinematic viscosity, $\beta$ ' is the coefficient of thermal expansion, $k$ is the turbulence kinetic energy per unit mass and arguments are as follows:

$$
\begin{gathered}
C D_{k w}=\max \left(2 \rho \frac{1}{\sigma_{\omega 2} \omega} \frac{\partial k}{\partial x_{j}} \frac{\partial \omega}{\partial x_{j}}, 1.0 \times 10^{-10}\right) \\
F_{2}=\tanh \left(\arg _{2}^{4}\right) \\
\arg _{2}=\max \left(\frac{2 \sqrt{k}}{\beta^{\prime} \omega y^{\prime}}, \frac{500 v}{y^{2} \omega}\right)
\end{gathered}
$$

\subsection{Numerical method}

A finite element method is used to solve the governing equations $(4) \sim(7)$. The propeller hydraulic turbine is comprised of 6 parts, which are divided into stationary and rotating zones. The rotating zone is applied to the MRF (Moving Reference Frame) model, in which individual cell zones are assigned different rotational and translational speeds to carry out steady-state approximations. Figure 3 shows the 3-D model of the propeller hydraulic turbine. The grid system of the modeled axial propeller turbine is depicted in Fig. 4. This grid system is modeled using the hybrid-grid system in which blade and guide vane parts were applied to the structured grid and the other parts were applied to the unstructured grid.

In order to facilitate the analysis of runner geometry effects, a numerical simulation was conducted for a partial periodic domain. The no-slip hydraulic boundary condition of velocity was adopted for the solid wall, while the inlet flow distribution was uniform for static pressure at the inflow duct. Opening-type inlet and outlet boundaries were considered to facilitate convergence.

\subsection{Grid system and boundary conditions}

The commercial code, CFX, has been used to solve the governing equations. It is based on the finite element method on a collocated grid. The grid systems of the hydraulic turbine given in Fig. 4 is adopted for the axial propeller turbine, with approximately $6.7 \times 10^{5}$ grids, of which $1.5 \times 10^{5}$ are in the guide vane zone, $9.6 \times 10^{4}$ in the runner zone, and $4.2 \times 10^{5}$ in the other stationary zone. This propeller hydraulic turbine is modeled using the hybrid grid system. Combining the unstructured (tetrahedral elements) and structured grid (hexahedral elements) significantly reduces the simulation time because the number of grid points to be re-meshed after each time step is restricted [1].

The boundary conditions of the axial propeller turbines are given in Table 1. The blade design is modeled under basic boundary conditions. The number of blades normally varies from 3 to 5 . The initial inlet boundary condition was set to $134.5 \mathrm{kPa}$ of atmospheric pressure in addition to the static pressure, which corresponds to $3.5 \mathrm{~m}$ of gross head. The atmospheric pressure was applied to the outlet boundary condition. Water was chosen as the working fluid for overall analysis, and the reference temperature was $25^{\circ} \mathrm{C}$.

The main parameters focused in this study were the rotational speed used and the gross head that changed $2.5 \sim 4 \mathrm{~m}$ as an independent control variable, while the mass flow rate, shaft power and turbine efficiency were used as dependent variables. 


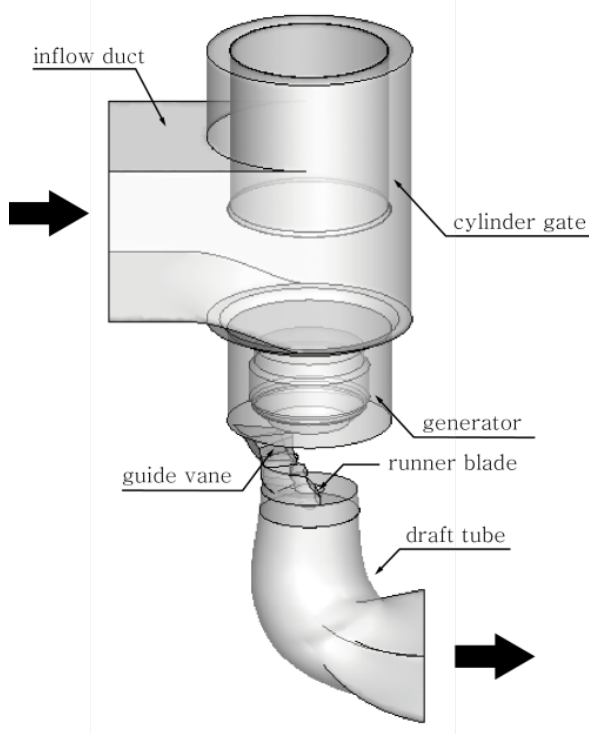

Fig. 3 3-D modeling of the axial propeller turbine applied to this study.

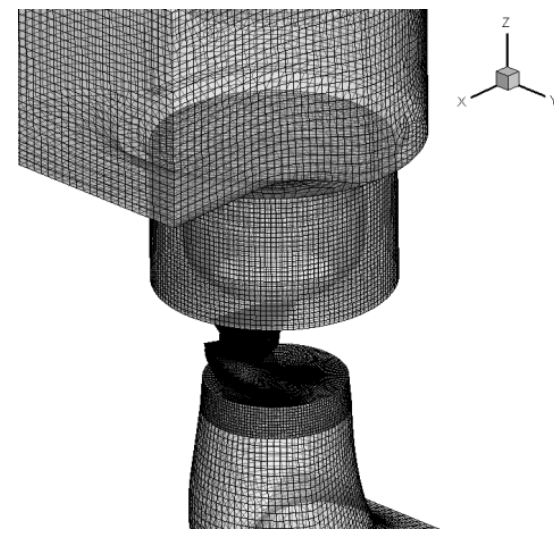

Fig. 4 Grid systems of the modeled axial propeller turbine.

Table 1. Details of the boundary conditions.

\begin{tabular}{ccc}
\hline No & Variable & Value \\
\hline 1 & Reference temperature & $298.15 \mathrm{~K}$ \\
2 & Density & $998 \mathrm{~kg} / \mathrm{m}^{3}$ \\
3 & Outlet pressure & $1 \mathrm{~atm}$ \\
4 & Initial rotational speed & $200 \mathrm{rpm}$ \\
5 & Initial gross head & $3.5 \mathrm{~m}$ \\
\hline
\end{tabular}

\section{Results and discussion}

In this paper, the non-dimensional group consisted of $\psi, \phi, P$, etc. , the so called head coefficient, flow coefficient, and power coefficient, respectively. It is useful to apply dimensionless terms in the analysis of a hydraulic turbine. Efficiency curves are compared in Figs. 5 and 6. In Fig. 5, the efficiency curve generally increases with increasing rotational speed but slightly decreases after the maximum point. On the other hand, in Fig. 6, the efficiency continuously increases with increasing gross head. The efficiency is rather influenced by the increases in head.

On the whole, the efficiency of the 4- or 5-blade hydraulic turbine was higher than that of the 3-blade hydraulic turbine. Thus, it is important to 4- or 5-blade design selection criteria to generate continuous electric power. Shaft power and efficiency curves are shown in Figs. 7 and 8 with respect to speed and gross head variations. Maximum power and efficiency are obtained at around $200 \mathrm{rpm}$ and $3.5 \mathrm{~m}$.

In order to validate the numerical method, we used the shaft power data with 5-blade which were obtained from one of the operating power plants in Korea. Comparison results are shown in Fig. 8 and showed good agreement. The average discrepancy of the shaft power between operating data and numerical results was given in the range of $8 \%$ at various head conditions.

As shown in Table 1, this study was conducted with the runner design based on the free vortex method and velocity triangles 
at rotational speeds of $200 \mathrm{rpm}$ and gross head of $3.5 \mathrm{~m}$. The hydraulic efficiency equation for an axial propeller turbine is given by Eq. (15).

$$
\eta_{\text {hyd }}=\frac{\text { hydraulic shaft head }}{\text { gross head }}=\frac{\Delta C_{x} \cdot U}{g \cdot H_{\text {gross }}}
$$

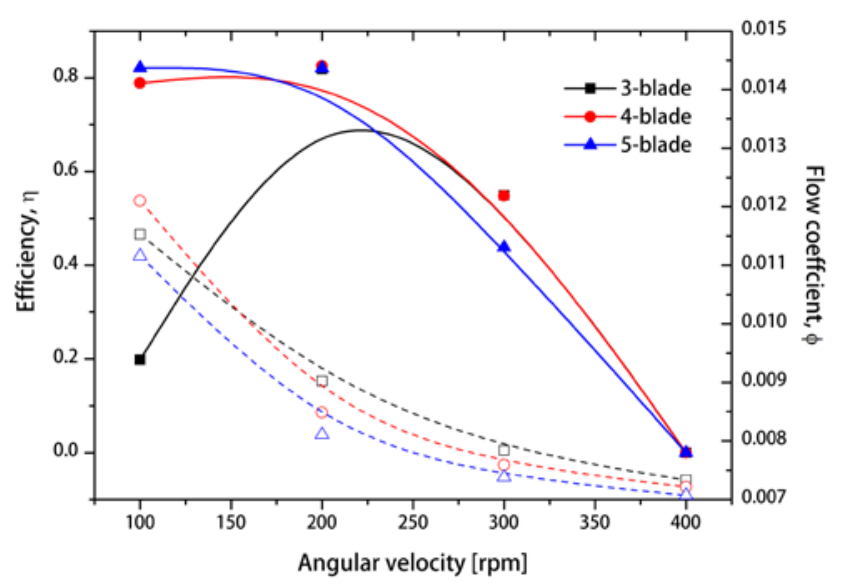

Fig. 5 Efficiency (solid lines) and flow coefficient, $\phi$ (dashed lines) for various values of angular velocity.

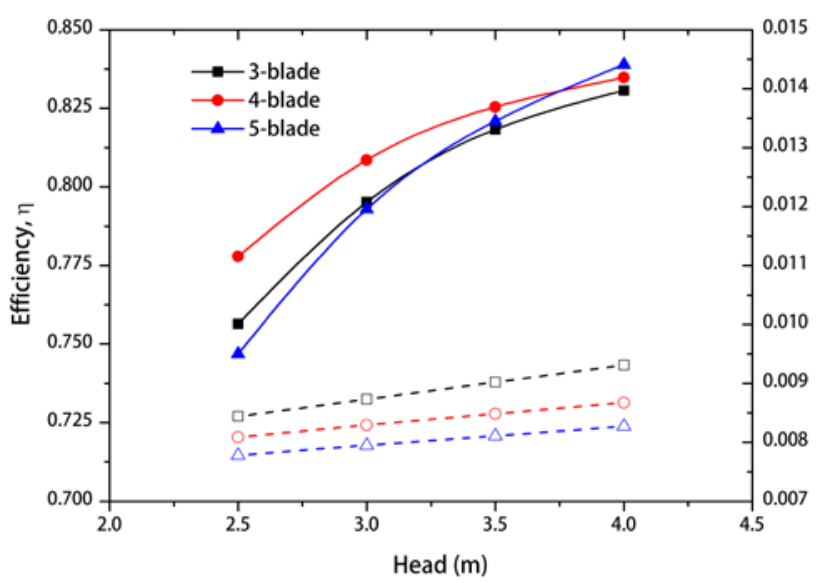

Fig. 6 Efficiency (solid lines) and flow coefficient, $\phi$ (dashed lines) for various values of head.

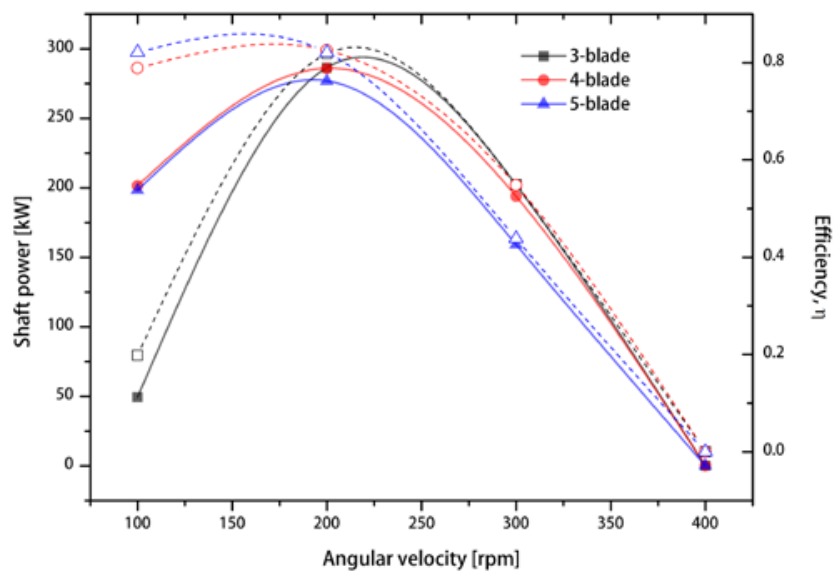

Fig. 7 Shaft power (solid lines) and efficiency (dashed lines) curves for various values of angular velocity.

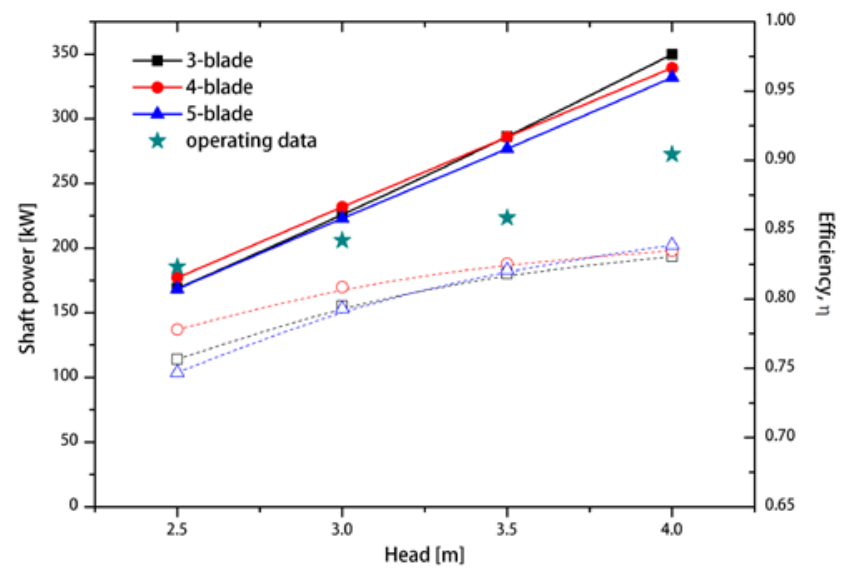

Fig. 8 Shaft power (solid lines) and efficiency (dashed lines) curves for various values of head. The symbol ( $\star$ ) denotes the operating data of the shaft power with 5-blade hydro turbine.

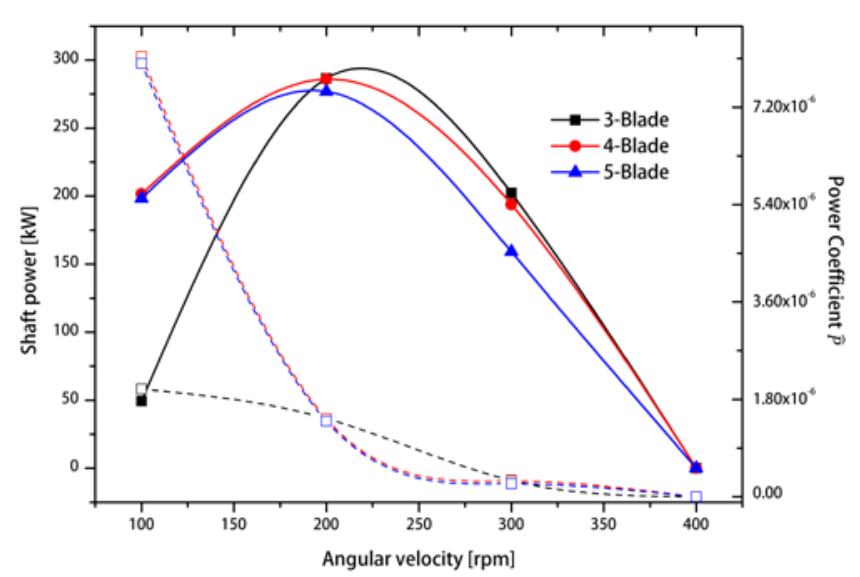

Fig. 9 Shaft power (solid lines) and power coefficient (dashed lines) curves for various values of angular velocity.

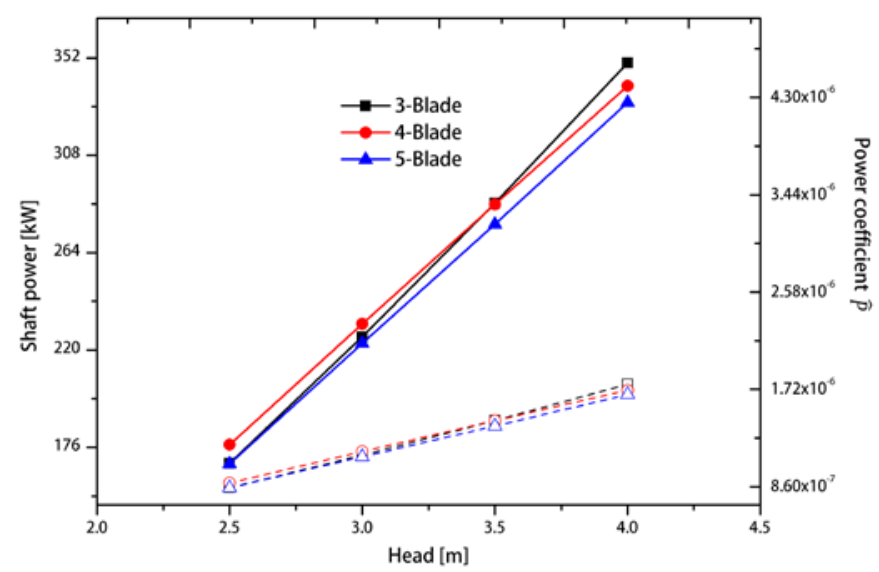

Fig. 10 Shaft power (solid lines) and power coefficient (dashed lines) curves for various values of head. 
When the gross head increases, the efficiency curves decrease past the maximum point because the increased gross head in Eq. (13) reduces the overall efficiency value. This study was conducted based on the practical model with blade number of 5 . The 4blade case showed a high efficiency, while the 3- or 5-blade case showed rapid changes in speed and head.

The results of shaft power and power coefficient are shown in Figs. 9 and 10. The performance characteristics compared in Fig. 10 show the decreasing trend in the power coefficient with increasing speed, although increasing gross head has a positive effect on the power coefficient.

\section{Conclusions}

This numerical study presented important and interesting results concerning flow hydraulics within a runner under variations of design parameters like the blade number. The 4-blade case is recommended to achieve high efficiency for wide ranges of gross head and speed. In the 3-blade case, the efficiency changed rapidly with increasing speed and gross head. In the 5-blade case, the efficiency was slightly lower than that of the 4-blade case since the increased blade number contributed to the increase in friction.

The flow coefficient increased in proportion to the efficiency with increasing gross head. The initial runner design was developed for a gross head of $3.5 \mathrm{~m}$ and speed of $200 \mathrm{rpm}$. Thus, other non-dimensional curves represented decreasing trends past the maximum point of $200 \mathrm{rpm}$ except the power coefficient curves. The power coefficient showed a decreasing trend with increasing speed but showed an increasing trend in proportion with the increase of the gross head. These results should be validated by experiments to identify any deficiencies in the non-dimensional analysis.

\section{Nomenclature}

$\begin{array}{ll}C & \text { Absolute velocity }[\mathrm{m} / \mathrm{s}] \\ d & \text { Hub diameter }[\mathrm{m}] \\ D & \text { Tip diameter }[\mathrm{m}] \\ g & \text { Acceleration due to gravity }\left[\mathrm{m} / \mathrm{s}^{2}\right] \\ H & \text { Head parameter }[\mathrm{m}] \\ N & \text { Rotational speed }[\mathrm{rev} / \mathrm{min}] \\ P & \text { Power }[\mathrm{kW}] \\ Q & \text { Discharge }\left[\mathrm{m}^{3} / \mathrm{s}\right] \\ r & \text { Radius }[\mathrm{m}] \\ T & \text { Torque }[\mathrm{N} \cdot \mathrm{m}] \\ U & \text { Tangential blade velocity }[\mathrm{m} / \mathrm{s}] \\ W & \text { Relative velocity }[\mathrm{m} / \mathrm{s}]\end{array}$

$\begin{array}{ll}\text { Greek symbols } & \\ \alpha & \text { Absolute flow angle }\left[^{\circ}\right] \\ \beta & \text { Relative flow of blade angle }\left[^{\circ}\right] \\ \mu & \text { Molecular viscosity }[\mathrm{kg} /(\mathrm{m} \cdot \mathrm{s})] \\ v & \text { Velocity }[\mathrm{m} / \mathrm{s}] \\ \omega & \text { Angular velocity }[\mathrm{rad} / \mathrm{s}] \\ \sigma & k-\omega \text { turbulent model constant } \\ \rho & \text { Density }\left[\mathrm{kg} / \mathrm{m}^{3}\right] \\ \eta & \text { Efficiency }[-] \\ \text { Subscripts } & \\ 1 & \text { Runner inlet } \\ 2 & \text { Runner exit } \\ r & \text { Axial direction }\end{array}$

\section{References}

[1] S.-S. Byeon and Y.-J. Kim, 2012, "Numerical study on the flow characteristics of the axial propeller hydro turbine," The Eighth Thermal and Fluids Engineering Conference, GSF26-018.

[2] Singh, P. and Nestmann, F., 2011, "Experimental investigation of the influence of blade height and blade number on the performance of low head axial flow turbines,” J. of Renewable Energy, Vol. 36, pp. 272-281.

[3] Singh, P. and Nestmann, F., 2009, “Experimental optimization of a free vortex propeller runner for micro hydro application,” J. of Renewable Energy, Vol. 33, pp. 991-1002.

[4] Singh, P. and Nestmann, F., 2010, "Exit blade geometry and part-load performance of small axial flow propeller turbine: An experimental investigation,” J. of Renewable Energy, Vol. 34, pp. 798-811.

[5] Yousef Yassi and Safar Hashemloo, 2010, "Improvement of the efficiency of the Agnew micro hydro turbine at part loads due to installing guide vanes mechanism,” Energy Conversion and Management, Vol. 51, pp. 1970-1975.

[6] L.M. C. Ferro, L. M. C. Gato, A.F. O. Falcao, 2011, "Design of the rotor blades of a mini hydraulic bulb-turbine,” Renewable Energy, Vol. 36, pp. 2395-2403.

[7] Alexander, K.V. Giddens, E. P., Fuller, A.M., 2009, “Axial-flow turbines for low head micro hydro systems,” J. of Renewable Energy, Vol. 34, pp. 35-47.

[8] Dixon, S. L., 2005, "Fluid Mechanics, Thermodynamics of Turbomachinery,” Elsevier, Oxford, UK, Chapters 2, 6 and 9.

[9] Gorla, R. S. R. and Khan, A. A., 2003, “Turbomachinery Design and Theory,” Marcel Dekker, New York, Chapters 1 and 3.

[10] Wilcox, D. C., 1986, “Multiscale model for turbulent flows,” AIAA 24th Aerospace Sciences Meeting, American Institute of Aeronautics and Astronautics, pp. 1598-1605.

[11] Menter, F. R., 1994, “Two-equation eddy-viscosity turbulence models for engineering applications,” AIAA-Journal, Vol. 32(8), pp. 1598-1605.

[12] ANSYS CFX ver.13 User’s Guide, 2011, ANSYS Inc. 


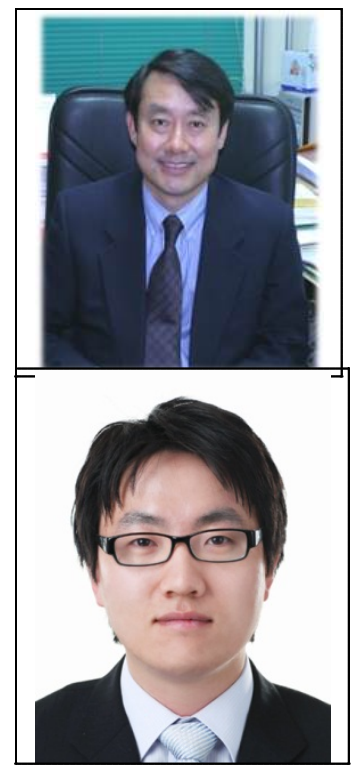

Youn-Jea Kim received his B.S. degree in Mechanical Engineering from Sungkyunkwan University, Korea, in 1982. He then received his M. S. and Ph.D. degrees from the State University of New York at Buffalo, USA in 1987 and 1990, respectively. Dr. Kim is currently a Professor at the School of Mechanical Engineering at Sunkyunkwan University in Korea. Dr. Kim's research interests include gas dynamics, MEMS and fluid-machineries, etc.

Sun-Seok Byeon received his B.S. degree in Mechanical Engineering from Sungkyunkwan University, Korea, in 2007. He then received his M.S. degrees from Sungkyunkwan University in 2013. Mr. Byeon is currently a candidate for the Ph.D degree at the Graduate School of Mechanical Engineering at Sunkyunkwan University in Korea. Mr. Byeon’s research interests include turbo-machineries. 